\title{
A breakpoint search approach for convex resource allocation problems with bounded variables
}

\author{
Anja De Waegenaere · Jacco L. Wielhouwer
}

Received: 3 June 2009 / Accepted: 25 January 2011 / Published online: 13 February 2011

(C) The Author(s) 2011. This article is published with open access at Springerlink.com

\begin{abstract}
We present an efficient approach to solve resource allocation problems with a single resource, a convex separable objective function, a convex separable resource-usage constraint, and variables that are bounded below and above. Through a combination of function evaluations and median searches, information on whether or not the upper- and lowerbounds are binding is obtained. Once this information is available for all upper and lower bounds, it remains to determine the optimum of a smaller problem with unbounded variables. This can be done through a multiplier search procedure. The information gathered allows for alternative approaches for the multiplier search which can reduce the complexity of this procedure.
\end{abstract}

Keywords Resource allocation · Multiplier search ·

Non-linear programming $\cdot$ Pegging

We thank Dick den Hertog and John Einmahl for helpful suggestions.

\footnotetext{
A. De Waegenaere $(\varangle)$

CentER for Economic Research and Department of Econometrics and Operations Research, Tilburg University, P.O. Box 90153, 5000 LE Tilburg, The Netherlands e-mail: a.m.b.DeWaegenaere@uvt.nl

J. L. Wielhouwer

Department of Accounting, Faculty of Economics, VU University Amsterdam, Room 2E-31, De Boelelaan 1105, 1081 HV Amsterdam, The Netherlands

e-mail: jwielhouwer@feweb.vu.nl
} 


\section{Introduction}

We focus on the continuous variable resource allocation problem of the following form:

$$
\begin{aligned}
& \min _{\left(d_{1}, \ldots, d_{N}\right)} \sum_{k=1}^{N} f_{k}\left(d_{k}\right) \\
& \text { s.t. } \\
& \quad \sum_{k=1}^{N} g_{k}\left(d_{k}\right) \leq D, \\
& \quad l_{k} \leq d_{k} \leq u_{k}, \quad \text { for } k=1, \ldots, N,
\end{aligned}
$$

where the performance functions $f_{k}(\cdot)$ and the resource-usage functions $g_{k}(\cdot)$ are differentiable and convex, and $-\infty<l_{k}<u_{k}<+\infty$. The operations research literature provides numerous examples of resource allocation problems of type (1) (see, e.g., $[9,10,15,17,18])$. In addition, efficient approaches for solving problem (1) are particularly important for applications that require solving a series of subproblems of type (1). Examples are heuristics and branch and bound algorithms for solving integer valued resource allocation problems (see, e.g., [5]), and algorithms for resource allocation problems with multiple resource constraints (see, e.g., [2,4,6, 19]).

In this paper we present a generalization of the breakpoint search approach for quadratic knapsack problems developed in [16], and extended by [12]. It is wellknown that optimization problems of type (1) can be solved through a multiplier search procedure. Specifically, by invoking the KKT conditions, all the variables can be expressed as a function of the Lagrange multiplier $\lambda$ of the resource-usage constraint, i.e. $d_{k}=d_{k}(\lambda)$. Then, either the resource usage constraint is not binding, and the optimal solution is given by $d_{k}^{*}=d_{k}(0)$, or the optimum is found by solving the equation $g(\lambda):=\sum_{k=1}^{N} g_{k}\left(d_{k}(\lambda)\right)=D$ in the unknown Lagrange multiplier $\lambda$ (see, e.g., $[2,3,13])$. In case of quadratic knapsack problems, the function $g(\lambda)$ is continuous, monotonic and piecewise linear. The idea of the breakpoint search approach then is to identify two consecutive breakpoints where the function $g(\cdot)$ has opposite sign. Then, $g(\lambda)$ is linear between these two breakpoints, and the optimal Lagrange multiplier is found through linear interpolation (see, e.g., $[7,8,11,12,16])$. We generalize this approach to convex resource allocation problems, with specific emphasis on how the Lagrange multiplier is found once the breakpoint search is completed, and how the breakpoint search yields information on whether or not bounds are binding. Specifically, the algorithm we present provides an efficient approach to classify each upper and lower bound as either binding or not binding without solving the resource allocation problem or a relaxation thereof. The information is obtained on the basis of function evaluations and median searches. Once every bound is classified, variables with a binding bound can be pegged at that bound, and it remains to solve a resource allocation problem with unbounded variables. In contrast to the quadratic case, however, the solution to this relaxed problem is not necessarily available in closed form expression. It can be solved through a multiplier search procedure, where the information on whether or not bounds are binding is used to: (i) determine tight bounds on the interval in which the optimal Lagrange multiplier can be found, and, (ii) identify a 
number of alternative equations that each yield the optimal Lagrange multiplier. In this way, the information gathered through function evaluations and rankings contributes to reducing the complexity of the multiplier search procedure for the relaxed problem. Compared to existing pegging and/or multiplier search methods for convex resource allocation problems, e.g., [1-4,13-15,20], an important advantage of the breakpoint search approach is that it does not require full ranking, and it does not require solving a series of relaxed problems. Solving a series of relaxed problems may be computationally demanding when no closed form solution exists. For a thorough review of algorithms for resource allocation problems, we refer to [17].

The paper is organized as follows. In Sect. 2, we first characterize the solution of optimization problem (1) in case some information is available on whether bounds are binding. Then, we show how function evaluations and ranking operations allow to identify all variables for which either the lower or the upper bound is binding. In Sect. 3 we use these results to present an efficient algorithm to solve the optimization problem. The paper is concluded in Sect. 4. All proofs are deferred to the Appendix.

\section{Identifying binding bounds}

Our goal in this paper is to present an efficient approach to solve optimization problem (1). The key idea is to gather information on whether or not bounds are binding. In this section we show how all variables for which the upper or lower bound is binding can be identified through a combination of function evaluations and median searches. First, in Theorem 1, we characterize the optimal solution of problem (1) in case some information is available on whether or not bounds are binding, and show how information on whether or not bounds are binding can substantially reduce the complexity of a multiplier search procedure to determine the optimum. Then, in Theorem 2, we show how information on whether or not bounds are binding can be obtained through specific function evaluations. First, we introduce the assumptions. Let us denote:

$$
F_{k}(d):=\frac{f_{k}^{\prime}(d)}{g_{k}^{\prime}(d)}, \quad k=1, \ldots, N
$$

\section{Assumptions}

$A_{1}$ For all $k, f_{k}(\cdot)$ and $g_{k}(\cdot)$ are continuously differentiable and convex;

$A_{2}$ for all $k, g_{k}(\cdot)$ is strictly monotone;

$A_{3}$ the functions $g_{k}\left(F_{k}^{-1}(\cdot)\right)$ for $k=1, \ldots, N$, are either all strictly increasing or all strictly decreasing on their domain.

Assumptions $A_{1}-A_{3}$ allow for a number of classes of problems. For example, they allow for the case where all resource usage functions $g_{k}(\cdot)$ are linear, but for some variables, the function $g_{k}(\cdot)$ is increasing, whereas for other variables, the function $g_{k}(\cdot)$ is decreasing. For notational convenience, we will focus in this paper on the case where:

$A_{4} g_{k}^{\prime}(\cdot)>0, \quad f_{k}^{\prime}(\cdot) \leq 0$, and $f_{k}^{\prime \prime}(\cdot)>0$ for all $k$ 
The main results in this paper, however, can easily be extended to any setting where assumptions $A_{1}-A_{3}$ are satisfied. Note that, in contrast to other multiplier search approaches (such as, e.g., $[2,3,13]$ ), we do not require that the nonlinear equations $f_{k}^{\prime}(\cdot)+\lambda g_{k}^{\prime}(\cdot)=0$, have a solution for all $k$ and all $\lambda \in \mathbb{R}^{+}$(or, equivalently, that $F_{k}^{-1}(-\lambda)$ exists for all $\left.\lambda \in \mathbb{R}^{+}\right)$.

Before we proceed, first note that if $\sum_{k=1}^{N} g_{k}\left(u_{k}\right) \leq D$, then it follows immediately from assumption $A_{4}$ that the optimal allocation is given by $d_{k}^{*}=u_{k}$ for all $k$. Therefore, we can assume without loss of generality that $\sum_{k=1}^{N} g_{k}\left(u_{k}\right)>D$, which implies that the resource-usage constraint is binding. Therefore, we present an approach to solve optimization problem (1) with the resource-usage constraint replaced by the equality constraint $\sum_{k=1}^{N} g_{k}\left(d_{k}\right)=D$.

As argued before, the main idea of the approach that we present in this paper is to identify, in subsequent iterations, all variables for which a bound is binding. We therefore introduce the following notation:

- $\mathcal{K}_{l b}$ is the set of variables for which it is known that the corresponding lower bound is binding,

- $\mathcal{K}_{u b}$ is the set of variables for which it is known that the corresponding upper bound is binding,

- $\mathcal{K}_{\text {lnb }}$ is the set of variables for which it is known that the corresponding lower bound is not binding,

- $\mathcal{K}_{\text {unb }}$ is the set of variables for which it is known that the corresponding upper bound is not binding,

- $\mathcal{K}_{b n b}$ is the set of variables for which it is known that both bounds are not binding, i.e., $\mathcal{K}_{\text {bnb }}=\mathcal{K}_{\text {lnb }} \cap \mathcal{K}_{\text {unb }}$.

The information will be summarized in $\mathcal{K}=\left\{\mathcal{K}_{l b}, \mathcal{K}_{u b}, \mathcal{K}_{\text {lnb }}, \mathcal{K}_{u n b}\right\}$. Now let

$$
\begin{aligned}
& \bar{\lambda}(\mathcal{K}):=\min \left\{\left\{F_{k}\left(l_{k}\right): k \in \mathcal{K}_{l b}\right\} \cup\left\{F_{k}\left(u_{k}\right): k \in \mathcal{K}_{u n b}\right\}\right\} \\
& \underline{\lambda}(\mathcal{K}):=\max \left\{\left\{F_{k}\left(l_{k}\right): k \in \mathcal{K}_{l n b}\right\} \cup\left\{F_{k}\left(u_{k}\right): k \in \mathcal{K}_{u b}\right\}\right\} .
\end{aligned}
$$

Then, we define the following function:

$$
\Psi(\lambda, \mathcal{K}):=D-\sum_{k=1}^{N} g_{k}\left(\xi_{k}(\lambda, \mathcal{K})\right), \quad \text { for } \lambda \in[\underline{\lambda}(\mathcal{K}), \bar{\lambda}(\mathcal{K})]
$$

where

$$
\begin{aligned}
\xi_{k}(\lambda, \mathcal{K}) & =F_{k}^{-1}\left(\min \left\{\max \left\{\lambda, F_{k}\left(l_{k}\right)\right\}, F_{k}\left(u_{k}\right)\right\}\right) & & \text { if } k \notin\left(\mathcal{K}_{l b} \cup \mathcal{K}_{u b} \cup \mathcal{K}_{l n b} \cup \mathcal{K}_{u n b}\right), \\
& =F_{k}^{-1}\left(\min \left\{\lambda, F_{k}\left(u_{k}\right)\right\}\right), & & \text { if } k \in \mathcal{K}_{l n b} \backslash \mathcal{K}_{\text {bnb }}, \\
& =F_{k}^{-1}\left(\max \left\{\lambda, F_{k}\left(l_{k}\right)\right\}\right), & & \text { if } k \in \mathcal{K}_{u n b} \backslash \mathcal{K}_{\text {bnb }}, \\
& =F_{k}^{-1}(\lambda), & & \text { if } k \in \mathcal{K}_{\text {bnb }}, \\
& =l_{k}, & & \text { if } k \in \mathcal{K}_{l b}, \\
& =u_{k}, & & \text { if } k \in \mathcal{K}_{u b} .
\end{aligned}
$$


Note that even though the domain of $F_{k}^{-1}(\cdot)$ may be a strict subset of $\mathbb{R}^{-}$, the functions $\xi_{k}(\lambda, \mathcal{K})$ are well-defined for $\lambda \in[\underline{\lambda}(\mathcal{K}), \bar{\lambda}(\mathcal{K})]$. Consider, for example, a $k \in$ $\mathcal{K}_{u n b} \backslash \mathcal{K}_{\text {bnb }}$. Then, we need to show that $F_{k}^{-1}\left(\max \left\{\lambda, F_{k}\left(l_{k}\right)\right\}\right)$ exists. First, if $\lambda \leq$ $F_{k}\left(l_{k}\right)$, then it holds that $\xi_{k}(\lambda, \mathcal{K})=F_{k}^{-1}\left(F_{k}\left(l_{k}\right)\right)=l_{k}$. Second, if $\lambda>F_{k}\left(l_{k}\right)$, then $\lambda<\bar{\lambda}(\mathcal{K})$ and $k \in \mathcal{K}_{u n b}$ imply that $\lambda<F_{k}\left(u_{k}\right)$. Since $F_{k}(\cdot)$ is continuous, this implies that $F_{k}^{-1}(\lambda) \in\left[l_{i}, u_{i}\right]$ exists, and $\xi_{k}(\lambda, \mathcal{K})=F_{k}^{-1}(\lambda)$. Similar arguments can be used to verify that $\xi_{k}(\lambda, \mathcal{K})$ is well-defined for all $k$ and all $\lambda \in[\underline{\lambda}(\mathcal{K}), \bar{\lambda}(\mathcal{K})]$. Thus, in contrast to other multiplier search approaches (such as, e.g., $[2,3,13]$ ), we do not require that $F_{k}^{-1}(\lambda)$ exists for all $\lambda \in \mathbb{R}^{-}$.

Now, invoking the KKT conditions on optimization problem (1) where all variables in $\mathcal{K}_{l b}\left(\mathcal{K}_{u b}\right)$ are pegged at their lower bound (upper bound), and for all variables in $\mathcal{K}_{\text {lnb }}\left(\mathcal{K}_{\text {unb }}\right)$ the lower bound (upper bound) constraint is removed, immediately yields the following result.

Theorem 1 Let $\mathcal{K}=\left\{\mathcal{K}_{l b}, \mathcal{K}_{u b}, \mathcal{K}_{\text {lnb }}, \mathcal{K}_{\text {unb }}\right\}$ be given. Then, the optimal solution to problem (1) satisfies:

$$
d_{k}^{*}=\xi_{k}\left(\lambda^{*}, \mathcal{K}\right), \quad \text { for all } k=1, \ldots, N,
$$

for any $\lambda^{*} \in[\underline{\lambda}(\mathcal{K}), \bar{\lambda}(\mathcal{K})]$ that solves $\Psi\left(\lambda^{*}, \mathcal{K}\right)=0$.

Let us now consider the following two extreme cases:

$$
\begin{aligned}
\mathcal{K}_{\text {null }} & =\{\varnothing, \varnothing, \varnothing, \varnothing\}, \\
\mathcal{K}_{\text {full }} & =\left\{\mathcal{K}_{l b}, \mathcal{K}_{u b}, \mathcal{K}_{\text {lnb }}, \mathcal{K}_{u n b}\right\} \text { such that } \mathcal{K}_{l b} \cup \mathcal{K}_{u b} \cup \mathcal{K}_{\text {bnb }}=\{1, \ldots, N\} .
\end{aligned}
$$

$\mathcal{K}=\mathcal{K}_{\text {null }}$ represents the situation where no information is available on whether or not bounds are binding. In contrast, $\mathcal{K}=\mathcal{K}_{\text {full }}$ represents the situation where all binding bounds are identified, so that it remains to determine the optimal value for the variables for which neither the upper nor the lower bound is binding, which allows to reduce the original optimization problem (1) to the smaller problem:

$$
\begin{aligned}
& \min \sum_{k \in \mathcal{K}_{b n b}} f_{k}\left(d_{k}\right) \\
& \text { s.t. } \\
& \sum_{k \in \mathcal{K}_{b n b}} g_{k}\left(d_{k}\right)=D-\sum_{k \in \mathcal{K}_{l b}} g_{k}\left(l_{k}\right)-\sum_{k \in \mathcal{K}_{u b}} g_{k}\left(u_{k}\right) .
\end{aligned}
$$

Identifying the set $\mathcal{K}_{\text {full }}$ therefore allows to replace the original optimization problem (1) by the simpler problem (7), and it follows from Theorem 1 that:

- For problem (1), $\mathcal{K}=\mathcal{K}_{\text {null }}$ so that

$$
d_{k}^{*}=F_{k}^{-1}\left(\max \left\{\min \left\{\lambda^{*}, F_{k}\left(u_{k}\right)\right\}, F_{k}\left(l_{k}\right)\right\}\right), \quad \text { for all } k=1, \cdots, N,
$$

with $\lambda^{*} \in \mathbb{R}$ such that

$$
\sum_{k=1}^{N} g_{k}\left(F_{k}^{-1}\left(\max \left\{\min \left\{\lambda^{*}, F_{k}\left(u_{k}\right)\right\}, F_{k}\left(l_{k}\right)\right\}\right)\right)=D
$$


- For problem (7), $\mathcal{K}=\mathcal{K}_{\text {full }}$ so that

$$
\begin{array}{ll}
d_{k}^{*}=F_{k}^{-1}\left(\lambda^{*}\right), & \text { for all } k \in \mathcal{K}_{b n b}, \\
d_{k}^{*}=l_{k}, & \text { for all } k \in \mathcal{K}_{l b}, \\
d_{k}^{*}=u_{k}, & \text { for all } k \in \mathcal{K}_{u b},
\end{array}
$$

with $\lambda^{*} \in\left[\underline{\lambda}\left(\mathcal{K}_{f u l l}\right), \bar{\lambda}\left(\mathcal{K}_{f u l l}\right)\right]$ such that

$$
\sum_{k \in \mathcal{K}_{b n b}} g_{k}\left(F_{k}^{-1}\left(\lambda^{*}\right)\right)=D-\sum_{k \in \mathcal{K}_{l b}} g_{k}\left(l_{k}\right)-\sum_{k \in \mathcal{K}_{u b}} g_{k}\left(u_{k}\right) .
$$

Comparing (8) and (9), we see that the information in $\mathcal{K}_{\text {full }}$ allows significant efficiency gains in multiplier searching, since, (i) the number of minimum and maximum terms reduces from $N$ to zero, (ii) $\lambda^{*}$ is known to be in $\left[\underline{\lambda}\left(\mathcal{K}_{f u l l}\right), \bar{\lambda}\left(\mathcal{K}_{f u l l}\right)\right]$, and, (iii) $F_{k}^{-1}(\lambda)$ only needs to be evaluated for $k \in \mathcal{K}_{b n b}$. The latter is particularly relevant in cases where (some) $F_{k}^{-1}(\lambda)$ are not available in closed form expression, so that every evaluation of $F_{k}^{-1}(\lambda)$ requires a numerical procedure.

The above clearly indicates that there is an advantage to identifying binding bounds. Theorem 2 shows how bounds can be classified as either binding or not binding through function evaluations and ranking operations.

Theorem 2 Let $\mathcal{K}=\left\{\mathcal{K}_{l b}, \mathcal{K}_{u b}, \mathcal{K}_{\text {lnb }}, \mathcal{K}_{u n b}\right\}$ and $\lambda \in[\underline{\lambda}(\mathcal{K}), \bar{\lambda}(\mathcal{K})]$ be given. Then,

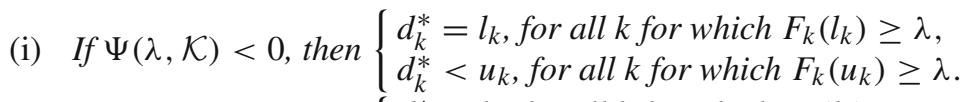

(ii) If $\Psi(\lambda, \mathcal{K})>0$, then $\left\{\begin{array}{l}d_{k}^{*}>l_{k}, \text { for all } k \text { for which } F_{k}\left(l_{k}\right) \leq \lambda \text {, } \\ d_{k}^{*}=u_{k} \text {, for all } k \text { for which } F_{k}\left(u_{k}\right) \leq \lambda \text {. }\end{array}\right.$

(iii) If $\Psi(\lambda, \mathcal{K})=0$, then $d_{k}^{*}=\xi_{k}(\lambda, \mathcal{K})$ for all $k=1, \ldots, N$.

The above theorem states that with each evaluation of $\Psi(\lambda, \mathcal{K})$ for some $\lambda$ and $\mathcal{K} \neq \mathcal{K}_{\text {full }}$, additional information can be obtained on whether or not certain bounds are binding. Specifically,

(i) if $\Psi(\lambda, \mathcal{K})<0$, it is known whether the lower bound (upper bound) is binding for every variable $k$ with $F_{k}\left(l_{k}\right) \geq \lambda\left(F_{k}\left(u_{k}\right) \geq \lambda\right)$

(ii) if $\Psi(\lambda, \mathcal{K})>0$, it is known whether the lower bound (upper bound) is binding for every variable $k$ with $F_{k}\left(l_{k}\right) \leq \lambda\left(F_{k}\left(u_{k}\right) \leq \lambda\right)$;

(iii) if $\Psi(\lambda, \mathcal{K})=0$, the optimum is given by $d_{k}^{*}=\xi_{k}(\lambda, \mathcal{K})$ for all $k=1, \ldots, N$.

How much information is obtained through evaluation of $\Psi(\lambda, \mathcal{K})$ clearly depends on the value of $\lambda$. Specifically, when $\lambda \in\left\{F_{k}\left(l_{k}\right): k \notin\left(\mathcal{K}_{l b} \cup \mathcal{K}_{\text {lnb }}\right)\right\}$ or $\lambda \in\left\{F_{k}\left(u_{k}\right)\right.$ : $\left.k \notin\left(\mathcal{K}_{u b} \cup \mathcal{K}_{u n b}\right)\right\}$, information is obtained about at least one bound. For example, if $\Psi\left(F_{i}\left(l_{i}\right), \mathcal{K}\right)<0$, it follows from Theorem 2(i) that the lower bound for variable $i$ is binding. It also follows that the lower bound for variable $k$ is binding for all $k$ with $F_{k}\left(l_{k}\right) \geq F_{i}\left(l_{i}\right)$, and that the upper bound for variable $k$ is not binding for all $k$ with $F_{k}\left(u_{k}\right) \geq F_{i}\left(l_{i}\right)$. This information allows to update $\mathcal{K}=\left\{\mathcal{K}_{l b}, \mathcal{K}_{u b}, \mathcal{K}_{\text {lnb }}, \mathcal{K}_{\text {unb }}\right\}$. 


\section{The algorithm}

In this section we present an algorithm that determines for each upper and lower bound whether or not it is binding, without actually solving the optimization problem, or a relaxation thereof. The main idea of the algorithm is to invoke Theorem 2 to update $\mathcal{K}$, starting from no information, i.e. $\mathcal{K}=\{\varnothing, \varnothing, \varnothing, \varnothing\}$ until $\mathcal{K}=\mathcal{K}_{\text {full }}$. Then, the optimum is found by solving (9).

\section{Algorithm}

Step 0: Set $\mathcal{K}=\{\varnothing, \varnothing, \varnothing, \varnothing\}$.

Step 1: Choose a breakpoint $\lambda \in \mathcal{U}:=\left\{F_{k}\left(l_{k}\right): k \notin\left(\mathcal{K}_{l b} \cup \mathcal{K}_{\operatorname{lnb}}\right)\right\} \cup\left\{F_{k}\left(u_{k}\right): k \notin\right.$ $\left.\left(\mathcal{K}_{u b} \cup \mathcal{K}_{u n b}\right)\right\}$.

Step 2: Compute $\Psi(\lambda, \mathcal{K})$.

Step 3: If $\Psi(\lambda, \mathcal{K})=0$, then STOP with $\lambda^{*}=\lambda$.

Step 4: If $\Psi(\lambda, \mathcal{K})<0$ then:

$-\mathcal{K}_{l b}=\mathcal{K}_{l b} \cup\left\{k \notin\left(\mathcal{K}_{l b} \cup \mathcal{K}_{l n b}\right): F_{k}\left(l_{k}\right) \geq \lambda\right\}$,

$-\mathcal{K}_{u n b}=\mathcal{K}_{u n b} \cup\left\{k \notin\left(\mathcal{K}_{u b} \cup \mathcal{K}_{u n b}\right): F_{k}\left(u_{k}\right) \geq \lambda\right\}$,

$-\bar{\lambda}=\lambda$.

Step 5: If $\Psi(\lambda, \mathcal{K})>0$, then:

- $\mathcal{K}_{u b}=\mathcal{K}_{u b} \cup\left\{k \notin\left(\mathcal{K}_{u b} \cup \mathcal{K}_{u n b}\right): F_{k}\left(u_{k}\right) \leq \lambda\right\}$,

$-\mathcal{K}_{\text {lnb }}=\mathcal{K}_{\text {lnb }} \cup\left\{k \notin\left(\mathcal{K}_{l b} \cup \mathcal{K}_{\text {lnb }}\right): F_{k}\left(l_{k}\right) \leq \lambda\right\}$,

$-\underline{\lambda}=\lambda$.

Step 6: Set $\mathcal{K}=\left\{\mathcal{K}_{l b}, \mathcal{K}_{u b}, \mathcal{K}_{\text {lnb }}, \mathcal{K}_{\text {unb }}\right\}$. If $\mathcal{K} \neq \mathcal{K}_{\text {full }}$, then go to Step 1. Else, go to Step 7.

Step 7: Find $\lambda^{*} \in[\underline{\lambda}, \bar{\lambda}]$ that satisfies (9).

In Step 1, the algorithm picks a breakpoint $\lambda=F_{k}\left(l_{k}\right)$ or $\lambda=F_{k}\left(u_{k}\right)$ corresponding to a bound for which it is not yet known whether it is binding. In Step 2, $\Psi(\lambda, \mathcal{K})$ is determined, where $\Psi(\cdot, \cdot)$ is as defined in (3), and Theorem 2 is applied to infer whether or not bounds are binding, i.e. to update the information set $\mathcal{K}$. This process is continued until either $\Psi(\lambda, \mathcal{K})=0$, or $\mathcal{K}=\mathcal{K}_{\text {full }}$.

The algorithm either immediately yields the optimum (if $\Psi(\lambda, \mathcal{K})=0$, in which case the optimum is given by $d_{k}^{*}=\xi_{k}(\lambda, \mathcal{K})$ for all $k$ ), or it yields the set $\mathcal{K}=\mathcal{K}_{\text {full }}$, i.e. all binding bounds are identified, so that it remains to determine the optimal value for the variables for which neither the upper nor the lower bound is binding. This implies that the original optimization problem (1) can be replaced by the smaller optimization problem (7). The latter can be solved through a multiplier search procedure, as in (9). The information obtained in Steps 1-6, however, has additional value in that it yields a number of alternatives for solving optimization problem (7) through multiplier searching, and provides a bounded interval in which the optimal Lagrange multiplier can be found in each case.

Theorem 3 The solution of (9) satisfies:

(i) $\lambda^{*} \in[\underline{\lambda}, \bar{\lambda}]$, where

$$
\begin{aligned}
& \bar{\lambda}=\min \left\{\left\{F_{k}\left(l_{k}\right): k \in \mathcal{K}_{l b}\right\} \cup\left\{F_{k}\left(u_{k}\right): k \in \mathcal{K}_{u n b}\right\}\right\}, \\
& \underline{\lambda}=\max \left\{\left\{F_{k}\left(l_{k}\right): k \in \mathcal{K}_{\text {lnb }}\right\} \cup\left\{F_{k}\left(u_{k}\right): k \in \mathcal{K}_{u b}\right\}\right\} .
\end{aligned}
$$


(ii) For any $i \in \mathcal{K}_{b n b}, \lambda^{*}=F_{i}\left(d_{i}^{*}\right)$, where $d_{i}^{*}$ satisfies

$$
\begin{gathered}
\sum_{k \in \mathcal{K}_{b n b} \backslash\{i\}} g_{k}\left(F_{k}^{-1}\left(F_{i}\left(d_{i}^{*}\right)\right)\right)+g_{i}\left(d_{i}^{*}\right)=D-\sum_{k \in \mathcal{K}_{l b}} g_{k}\left(l_{k}\right)-\sum_{k \in \mathcal{K}_{u b}} g_{k}\left(u_{k}\right), \\
d_{i}^{*} \in\left[F_{i}^{-1}(\underline{\lambda}), F_{i}^{-1}(\bar{\lambda})\right] \subset\left[l_{i}, u_{i}\right] .
\end{gathered}
$$

The above theorem shows that there are $\# \mathcal{K}_{b n b}+1$ alternatives to determine the optimal $d_{k}^{*}$, for all $k \in \mathcal{K}_{b n b}$ : solve (9), or pick any $i \in \mathcal{K}_{b n b}$ and solve (10). The latter has the advantage that it does not require evaluations of $F_{i}^{-1}(\cdot)$, which may be computationally intensive if the inverse is not available in closed form expression. For each alternative, the theorem provides an upper and a lower bound to the interval in which the solution can be found.

Let us finally discuss the computational efficiency of the algorithm. Patriksson [17] provides an extensive survey of algorithms for continuous non-linear resource allocation problems, and distinguishes two classes: pegging algorithms $[1,4,13,14,20]$ and multiplier search algorithms $[2,3,13,15] .{ }^{1}$ Our approach falls into the class of multiplier search approaches. As suggested by Patriksson [17], multiplier search approaches are to be preferred to pegging approaches in cases where the solution of equations of type (9) is not available in closed form expression. ${ }^{2}$ The multiplier search approaches have in common that they all require, in a last step, to determine $\lambda^{*}$ that solves (9). The computational efficiency of these approaches therefore depends on:

1. The efficiency of steps prior to solving (9). It holds that:

(a) When in Step $1, \lambda$ is chosen as the median of $\mathcal{U}$, then the number of elementary operations as well as the number of evaluations of $g_{k}(\cdot)$ and $F_{k}^{-1}(\cdot)$ in Steps 1-6 is $O(N \log N) .^{3}$ To the best of our knowledge, multiplier search algorithms for general convex resource allocation problems with lower complexity order do not exist.

(b) In contrast to existing multiplier search approaches (e.g., [2,3,13,15]), our algorithm does not require sorting. As argued also by Patriksson [17], sorting should be avoided unless one needs to solve several similar problems.

2. The efficiency of finding $\lambda^{*}$ such that $\Psi\left(\lambda^{*}\right)=0$ (Step 7). In our case, $\lambda^{*}$ can be determined by solving (9). Other existing multiplier search approaches for convex problems (e.g., $[2,3,13,15])$ require the same equation to be solved. However, finding $\lambda^{*}$ in our case can be done more efficiently because, as shown in Theorem 3 , the algorithm yields a number of alternatives for the equation that needs to be solved.

\footnotetext{
1 We focus on algorithms for general convex resource allocation problems. We do not consider approaches developed for specific objective functions only, such as, e.g., quadratic knapsack problems.

2 In contrast to multiplier search approaches, pegging approaches (e.g., [1,4,14,20]) require an equation of the form (9) (with different values for $\mathcal{K}_{l b}, \mathcal{K}_{u b}$, and $\mathcal{K}_{l n b}$ ) to be solved in every iteration.

3 Selecting $\lambda$ as the median of $\mathcal{U}$ requires $O(|\mathcal{U}|)$ operations. Since $\mathcal{U}$ is at least halved in each iteration, the algorithm requires $O(\log N)$ iterations (see, e.g., [12,16]). Determining $\Psi(\lambda)$ in Step 2 requires $O(N)$ elementary operations and $O(N)$ evaluations of $g_{k}(\cdot)$ and of $F_{k}^{-1}(\cdot)$.
} 
To summarize, as suggested by Patriksson [17], multiplier search approaches are to be preferred to pegging approaches in cases where the solution of equations of type (9) is not available in closed form expression. Moreover, within the class of multiplier search approaches, we expect that our algorithm will yield efficiency gains for problems for which running times are determined primarily by the computational effort in finding $\lambda^{*}$ such that $\Psi\left(\lambda^{*}\right)=0$. This is likely to be the case when the number of variables is relatively low (i.e., $N$ is small), but finding $\lambda^{*}$ is computationally intensive because every single evaluation of $\Psi(\cdot)$ requires determining $F_{i}^{-1}(\cdot)$ numerically for at least one variable $i \in \mathcal{K}_{b n b}$. In such cases, our approach allows to avoid determining $F_{i}^{-1}(\cdot)$ for an $i \in \mathcal{K}_{b n b}$, by solving (10) instead of (9).

\section{Conclusion}

In this paper we present an efficient approach for solving resource allocation problems with bounded variables, a separable convex objective function and a separable convex resource-usage constraint. The main idea of the approach is to obtain information on whether or not bounds are binding through function evaluations and ranking operations. Once this information is available for each bound, it remains to solve a resource allocation problem with unbounded variables. This relaxed problem is solved through a multiplier search procedure, where the information on whether or not bounds are binding is used to reduce the numerical complexity of root searching in two ways. First, it allows to determine tight bounds on the interval in which the optimal Lagrange multiplier can be found. Second, it allows to identify a number of alternative equations that each yield the optimal Lagrange multiplier. For future research it might be interesting to investigate the extent to which the information acquisition described in this paper can be useful in settings where resource allocation problems need to be solved repeatedly, such as, e.g., branch and bound approaches to solve integer valued resource allocation problems.

Open Access This article is distributed under the terms of the Creative Commons Attribution Noncommercial License which permits any noncommercial use, distribution, and reproduction in any medium, provided the original author(s) and source are credited.

\section{Appendix}

Proof of Theorem 1 Given that the resource-usage constraint is binding, and that it is known that for all variables in $\mathcal{K}_{\ln b}\left(\mathcal{K}_{u n b}\right)$ the lower (upper) bound is not binding, and that for all variables in $\mathcal{K}_{l b}\left(\mathcal{K}_{u b}\right)$ the lower (upper) bound is binding, the optimal value for all variables $d_{k}, k \in \mathcal{S}:=\{1, \ldots, N\} \backslash\left(\mathcal{K}_{l b} \cup \mathcal{K}_{u b}\right)$ can be found by solving the following optimization problem:

$$
\min _{\left\{d_{k}: k \in \mathcal{S}\right\}} \sum_{k \in \mathcal{S}} f_{k}\left(d_{k}\right)
$$




$$
\begin{array}{ll}
\text { s.t. } & \sum_{k \in \mathcal{S}} g_{k}\left(d_{k}\right)=D-\sum_{k \in \mathcal{K}_{l b}} g_{k}\left(l_{k}\right)-\sum_{k \in \mathcal{K}_{u b}} g_{k}\left(u_{k}\right), \\
& l_{k} \leq d_{k} \leq u_{k}, \quad \text { for } k \in \mathcal{S} \backslash\left(\mathcal{K}_{\text {lnb }} \cup \mathcal{K}_{u n b}\right) \\
& d_{k} \geq l_{k}, \quad \text { for } k \in \mathcal{S} \cap\left(\mathcal{K}_{u n b} \backslash \mathcal{K}_{\text {lnb }}\right) \\
& d_{k} \leq u_{k}, \quad \text { for } k \in \mathcal{S} \cap\left(\mathcal{K}_{\text {lnb }} \backslash \mathcal{K}_{\text {unb }}\right)
\end{array}
$$

The proof is similar to the proof in [2] for the case where $\mathcal{K}_{l b}=\mathcal{K}_{u b}=\mathcal{K}_{\text {lnb }}=$ $\mathcal{K}_{\text {unb }}=\varnothing$.

Proof of Theorem 2 Since $g_{k}^{\prime}(\cdot)>0$, the necessary and sufficient conditions for an optimum of problem (12) are as follows: there exist $\mu_{k} \geq 0, \tau_{k} \geq 0$, and $\lambda \leq 0$, such that

$$
\begin{cases}F_{k}\left(d_{k}\right)-\lambda-\mu_{k}+\tau_{k}=0, & k \in \mathcal{S}, \\ \mu_{k}\left(d_{k}-l_{k}\right)=0, & k \in \mathcal{S} \backslash \mathcal{K}_{\text {lnb }}, \\ \mu_{k}=0 & k \in \mathcal{S} \cap \mathcal{K}_{\text {lnb }} \\ \tau_{k}\left(d_{k}-u_{k}\right)=0, & k \in \mathcal{S} \backslash \mathcal{K}_{u n b}, \\ \tau_{k}=0 & k \in \mathcal{S} \cap \mathcal{K}_{u n b} \\ \sum_{k \in \mathcal{S}} g_{k}\left(d_{k}\right)=D-\sum_{k \in \mathcal{K}_{l b}} g_{k}\left(l_{k}\right)-\sum_{k \in \mathcal{K}_{u b}} g_{k}\left(u_{k}\right), & \\ l_{k} \leq d_{k} \leq u_{k}, & k \in \mathcal{S} \backslash\left(\mathcal{K}_{\text {lnb }} \cup \mathcal{K}_{u n b}\right), \\ d_{k} \geq l_{k}, & k \in \mathcal{S} \cap\left(\mathcal{K}_{u n b} \backslash \mathcal{K}_{\text {lnb }}\right), \\ d_{k} \leq u_{k}, & k \in \mathcal{S} \cap\left(\mathcal{K}_{\text {lnb }} \backslash \mathcal{K}_{u n b}\right) .\end{cases}
$$

(i) Let $\lambda \in \mathbb{R}, \mathcal{K}$, and $k \in\{1, \ldots, N\}$ be such that $\Psi(\lambda, \mathcal{K})<0$, and $F_{k}\left(l_{k}\right) \geq \lambda$. Now note that assumption $A_{4}$ implies that, for any $\mathcal{K}$, the function $\Psi(\cdot, \mathcal{K})$ is decreasing. Therefore,

$$
\Psi\left(F_{k}\left(l_{k}\right), \mathcal{K}\right) \leq \Psi(\lambda, \mathcal{K})<0 .
$$

Now suppose the lower bound for variable $k$ is not binding. Then there exists a solution $\mu_{k}^{*} \geq 0, \tau_{k}^{*} \geq 0$, and $\lambda^{*} \leq 0$ of (13) for which $\mu_{k}^{*}=0$, and

$$
\lambda^{*}=F_{k}\left(d_{k}^{*}\right)+\tau_{k}^{*}
$$

The fact that $\Psi(\cdot, \mathcal{K})$ is decreasing, $F_{k}(\cdot)$ is strictly increasing, $d_{k}^{*} \geq l_{k}, \tau_{k}^{*} \geq 0$, and $\Psi\left(\lambda^{*}, \mathcal{K}\right)=0$ then implies that

$$
\Psi\left(F_{k}\left(l_{k}\right), \mathcal{K}\right) \geq \Psi\left(F_{k}\left(d_{k}^{*}\right), \mathcal{K}\right)=\Psi\left(\lambda^{*}-\tau_{k}^{*}, \mathcal{K}\right) \geq \Psi\left(\lambda^{*}, \mathcal{K}\right)=0
$$

Therefore, by contradiction, it must hold that $d_{k}^{*}=l_{k}$.

Next, let $\lambda \in \mathbb{R}, \mathcal{K}$, and $k \in\{1, \ldots, N\}$ be such that that $\Psi(\lambda, \mathcal{K})<0$ and $F_{k}\left(u_{k}\right) \geq \lambda$. Then, $\Psi\left(F_{k}\left(u_{k}\right), \mathcal{K}\right) \leq \Psi(\lambda, \mathcal{K})<0=\Psi\left(\lambda^{*}, \mathcal{K}\right)$, so that $\lambda^{*}<F_{k}\left(u_{k}\right)$. Now suppose that $d_{k}^{*}=u_{k}$. Then (14) and $\tau_{k}^{*} \geq 0$ implies that $\lambda^{*} \geq F_{k}\left(u_{k}\right)$. Therefore, we can conclude that $d_{k}^{*}<u_{k}$.

(ii) Goes along the same lines as the proof of (i).

(iii) Follows immediately from Theorem 1. 
Proof of Theorem $3 \quad$ (i) Since $d_{k}^{*}=l_{k}$ for all $k \in \mathcal{K}_{l b}$, Theorem 2 with $\mathcal{K}=\mathcal{K}_{\text {full }}$ and $\lambda=F_{k}\left(l_{k}\right)$ implies that $\Psi\left(F_{k}\left(l_{k}\right), \mathcal{K}_{f u l l}\right) \leq 0$ for all $k \in \mathcal{K}_{l b}$. Since $\Psi\left(\lambda^{*}, \mathcal{K}_{\text {full }}\right)=0$, and $\Psi\left(\cdot, \mathcal{K}_{\text {full }}\right)$ is strictly decreasing, this implies that $\lambda^{*} \leq$ $F_{k}\left(l_{k}\right)$ for all $k \in \mathcal{K}_{l b}$. Similarly, it can be shown that $\lambda^{*} \leq F_{k}\left(u_{k}\right)$ for all $k \in \mathcal{K}_{\text {unb }}$, so that $\lambda^{*} \leq \bar{\lambda}$. The proof for $\lambda^{*} \geq \underline{\lambda}$ goes along the same lines.

(ii) It follows from (13) that $\lambda^{*}=F_{i}\left(d_{i}^{*}\right)$ for any $i \in \mathcal{K}_{b n b}$. Substituting this in (9) immediately implies that

$$
\sum_{k \in \mathcal{K}_{b n b} \backslash\{i\}} g_{k}\left(F_{k}^{-1}\left(F_{i}\left(d_{i}^{*}\right)\right)\right)+g_{i}\left(d_{i}^{*}\right)=D-\sum_{k \in \mathcal{K}_{l b}} g_{k}\left(l_{k}\right)-\sum_{k \in \mathcal{K}_{u b}} g_{k}\left(u_{k}\right) .
$$

Moreover, since $F_{i}(\cdot)$ is strictly increasing, $\lambda^{*} \in[\underline{\lambda}, \bar{\lambda}]$ implies $d_{i}^{*} \in\left[F_{i}^{-1}(\underline{\lambda})\right.$, $\left.F_{i}^{-1}(\bar{\lambda})\right]$

This concludes the proof.

\section{References}

1. Bitran, G.R., Hax, A.C.: Disaggregation and resource allocation using convex knapsack problems with bounded variables. Manag. Sci. 27, 431-441 (1981)

2. Bretthauer, K.M., Shetty, B.: The nonlinear resource allocation problem. Oper. Res. 43, 670-683 (1995)

3. Bretthauer, K.M., Shetty, B.: The nonlinear knapsack problem-algorithms and applications, invited review. Eur. J. Oper. Res. 138, 459-472 (2002)

4. Bretthauer, K.M., Shetty, B.: A pegging algorithm for the nonlinear resource allocation problem. Comput. Oper. Res. 29, 505-527 (2002)

5. Bretthauer, K.M., Shetty, B., Syam, S.: A projection method for the integer quadratic knapsack problem. J. Oper. Res. Soc. 47, 457-462 (1996)

6. Bretthauer, K.M., Shetty, B., Syam, S., Vokurka, R.J.: Production and inventory management under multiple resource constraints. Math. Comput. Model. 44, 85-95 (2006)

7. Brucker, P.: An O(n) algorithm for quadratic knapsack problems. Oper. Res. Lett. 3, 163-166 (1984)

8. Calamai, P.H., More, J.J.: Quasi-Newton updates with bounds. SIAM J. Numer. Anal. 24, 1434-1441 (1987)

9. Chao, X., Liu, L., Zheng, S.: Resource allocation in multisite service systems with intersite customer flows. Manag. Sci. 49(12), 1739-1752 (2003)

10. De Waegenaere, A., Wielhouwer, J.L.: Optimal tax depreciation lives and charges under regulatory constraints. OR Spectrum 24(2), 151-177 (2002)

11. Helgason, R., Kennington, J., Lall, H.: A polynomially bounded algorithm for a singly constrained quadratic program. Math. Program. 18, 338-343 (1980)

12. Kiwiel, K.C.: Breakpoint searching algorithms for the continuous quadratic knapsack problem. Math. Program. 112, 473-491 (2008)

13. Kodialam, M.S., Luss, H.: Algorithms for separable nonlinear resource allocation problems. Oper. Res. 46, 272-284 (1998)

14. Luss, H., Gupta, S.K.: Allocation of effort resources among competing activities. Oper. Res. 23, 360-366 (1975)

15. Nielsen, S.S., Zenios, S.A.: Massively parallel algorithms for singly constrained convex problems. ORSA J. Comput. 4(2), 166-181 (1992)

16. Pardalos, P.M., Kovoor, N.: An algorithm for a singly constrained class of quadratic programs subject to upper and lower bounds. Math. Program. 46, 321-328 (1990)

17. Patriksson, M.: A survey on the continuous nonlinear resource allocation problem. Eur. J. Oper. Res. 185, 1-46 (2008)

18. Shanthikumar, J.G., Xu, H.: Asymptotically optimal routing and service rate allocation in a multiserver queueing system. Oper. Res. 45(3), 464-469 (1997) 
19. Ventura, J.A., Weng, M.X.: Minimizing single-machine completion-time variance. Manag. Sci. 41, 1448-1455 (1995)

20. Zipkin, P.H.: Simple ranking methods for allocation of one resource. Manag. Sci. 26, 34-43 (1980) 